\title{
Regulierung personenbezogener Internet-Veröffentlichungen
}

Seit Jahren ist das Problem bekannt, doch die Politik wollte bisher nicht nachbessern: Das mit seiner Systematik aus den 90er Jahren stammende BDSG gibt keine Antwort auf die Frage, welche personenbezogenen Daten unter welchen Voraussetzungen im Internet veröffentlicht werden dürfen. Die Anprangerung à la „Rotten Neighbor" war ebenso wenig Anlass zum Handeln wie das ungewollte Erschließung von Personendaten über Suchmaschinen. Identitätsdiebstahl in Social Communities oder diffamierende Blogs lösten bei der Politik nur hilfloses Achselzucken aus. Auch Gerichtsentscheidungen blieben zunächst folgenlos, etwa die des Bundesverfassungsgerichts zum Schuldnerspiegel (BVerfG NJW 2002, 741) oder die Lindqvist-Entscheidung des Europäischen Gerichtshofes (EuGH JZ 2004, 242). Konkrete Regulierungsvorschläge (Weichert DuD 2009, 7) erfreuten sich keiner öffentlichen Wahrnehmung.

Dann kam die Auseinandersetzung zu Google Street View, ausgehend 2008 vom kommunalen Widerstand Molfsees, einem kleinen Ort bei Kiel, die bald die ganze Republik bewegte. Darf Google das persönliche Lebensumfeld bildlich im Internet darstellen? Als gäbe es nichts Sensibleres als Bilder von Hausfassaden! Jedenfalls löste der Konflikt sehr bürokratisch daher kommende Gesetzgebungsaktivitäten des Bundesrates aus (Entwurf zur BDSG-Änderung vom 18.08.2010, BT-Drs. 17/2765). Parallel entschied der Bundesgerichtshof, dass die anonyme Bewertung von Lehrern im Internet vom Grundrecht auf Meinungsfreiheit abgedeckt ist und deshalb z.B. \$29 Abs. 2 BDSG in verfassungskonformer Weise einfach nicht angewendet werden dürfe (BGH DuD 2009, 565).

Derart unter Handlungsdruck, lud die Bundesregierung am 20.09.2010 zu einem „Datenschutzgipfel“ und forderte dort von der Internetwirtschaft, sich durch Selbst- regulierung in Form eines DatenschutzKodexes selbst Grenzen zu setzen. Anlässlich des IT-Gipfels am 07.12.2010 wurde weiterhin ein rote Linien ziehender $\mathrm{Ge}$ setzentwurf angekündigt.

Angesichts dieser Diskussionszuspitzung und der Ungewissheit, was bei wirtschaftlicher Selbst- und regierungsseitiger Gesetzesregulierung herauskommen würde, sah sich das Unabhängige Landeszentrum für Datenschutz (ULD) veranlasst, am 27.10.2010 einen eigenen Gesetzentwurf als Diskussionsgrundlage und Blaupause vorzulegen, mit dem nicht nur die Veröffentlichung von Geodaten, sondern von Personendaten jeder Form im Netz einer rechtssicheren und zugleich entwicklungsoffenen Regulierung zugeführt werden soll (https://www.datenschutzzentrum.de/presse/20101027-gesetzesvorschlag-internet-regulierung.htm).

Im Zentrum des ULD-Vorschlags steht die Einführung eines $\$ 29$ a BDSG, der die elektronische „Veröffentlichung“von personenbezogenen Daten von einer Abwägung zwischen der Meinungsfreiheit nach Art. 5 GG und dem Datenschutz abhängig macht. Zwecks beschleunigter Konfliktregelung sind digitale Beschwerde- und Prüfungsrechte vorgesehen. Folgende weitere Punkte sollen geregelt werden:

- Verantwortlichkeit von inländischen Konzernunternehmen bei einem Sitz der Internetfirma außerhalb Europas,

- Abgrenzung von reinen Sachdaten zu personenbezogenen Daten,

- Anpassung der Definition der „verantwortlichen Stelle" an die Regelungen des Telemediengesetzes (TMG),

- Einführung des Prinzips „Privacy by Default", also der Pflicht zu datenschutzfreundlichen Grundeinstellungen, für Diensteanbieter,

- Regelung der elektronischen Einwilligung.
Der neue $₫ 29$ a verlangt von jedem Menschen wie jeder Stelle bei Bereitstellung personenbezogener Daten zum Abruf im Internet eine Abwägung zwischen Veröffentlichungsinteresse und den schutzwürdigen Interessen des oder der Betroffenen. Ein Überwiegen letzterer wird bei besonderen Arten personenbezogener Daten nach $₫ 3$ Abs. 9 BDSG unterstellt. $\mathrm{Da}$ an einer Abwägung kein Weg vorbei geht, hierfür aber keine spezifischen Abwägungskriterien festgelegt werden können, da die Fallkonstellationen so vielfältig wie das Internet sind, wählt der Entwurf einen prozeduralen Weg der Konfliktlösung: Widerspricht der Betroffene, so muss der Impressumspflichtige nach dem TMG umgehend reagieren. Tut er dies nicht, so wendet sich die Rechtmäßigkeitsvermutung gegen die Veröffentlichung. Ist ein Widerspruch aus einer allgemein zugänglichen Quelle erkennbar, so muss dieser beachtet werden (vgl. bisher $\S$ 29 Abs. 3 BDSG). Um zumindest bei Massenverarbeitungen à la Street View den Betroffenen vorab ein Chance auf Widerspruch zu geben, wird eine Benachrichtigung auf einer Internetseite Pflicht. Zudem soll eine ursprüngliche Datenquelle durch Versehen eines personenbezogenen Datums mit einem Meta-Löschdatum eine Chance zur Realisierung der „Gnade des Vergessens“ erhöht werden. An einer behördlichen Konfliktlösung durch die Datenschutzaufsicht, die natürlich in einem hohen Maße Opportunität walten lassen muss, geht kein Weg vorbei. Die Diskussion über diese Vorschläge wird zeigen, ob dieses Regelungskonzept umfassend genug und praktikabel ist. Gefordert sind jetzt die Politik und die Datenschutz-Community.

Thilo Weichert

Unabhängiges Landeszentrum für Datenschutz Schleswig-Holstein 